\title{
Ocurrencia, grado de afectación y controlabilidad de los eventos vitales en el desarrollo de la psicopatología: ¿impacto ambiental o consecuencia de una personalidad vulnerable?
}

\author{
ANDREA Cuevas CARDa \\ al260587@uji.es \\ M. ${ }^{a}$ AMPARO PÉREZ BARBERÁ \\ al189629@uji.es \\ LAURA MEZQUITA GUILLAMÓN \\ Imezquit@uji.es
}

\section{Resumen}

Introducción: La personalidad y los eventos vitales influyen en el desarrollo de psicopatología. No obstante, es posible que la afectación y ocurrencia de los eventos vitales no sea casual, sino que esté influenciada por la personalidad. Por ejemplo, el neuroticismo se ha asociado con la psicopatología internalizante y con informar de un mayor número y afectación de los eventos vitales. La impulsividad se ha asociado con los síntomas externalizantes y con sufrir más eventos controlables. El presente estudio evalúa en qué medida los eventos vitales influyen en el desarrollo de la psicopatología, una vez se controla el efecto de la personalidad. Método: En el 2004, 336 jóvenes (edad media $=21,68$, DT $=3,85 ; 61,3 \%$ mujeres) completaron el cuestionario de personalidad NEO-FFI. Cinco años más tarde rellenaron la escala de eventos vitales ISV y varios cuestionarios sobre síntomas internalizantes y externalizantes. Para alcanzar el objetivo, se realizaron análisis de regresión por pasos. Resultados: El neuroticismo, la responsabilidad, el número de eventos negativos y la afectación predijeron la sintomatología internalizante; mientras que la baja amabilidad, el neuroticismo, los eventos negativos y controlables y la afectación predijeron la sintomatología externalizante. Todos los efectos se mantuvieron significativos cuando se controló la personalidad, a excepción de los eventos controlables. Discusión: Los resultados indican que, por lo general, el efecto de los eventos vitales en la psicopatología no se produce únicamente por la influencia de la personalidad, sino que estos tendrían un impacto directo en el desarrollo de la psicopatología.

Palabras clave: personalidad, eventos vitales, psicopatología internalizante y externalizante.

\section{Abstract}

Introduction: Personality and life events influence the development of psychopathology. However, the involvement and occurrence of life events may not be accidental, but 
influenced by personality. For example, neuroticism has been associated with both internalising psychopathology (e.g., depression) and reporting more involvement of life events. Impulsivity has been associated with externalising symptoms (e.g., drug consumption) and with experiencing more controllable events (e.g., imprisonment). This study assesses to what extent life events influence the development of psychopathology once the effect of personality is controlled for. Method: In 2004, 336 young people (mean age $=21.68, \mathrm{SD}=3.85$; women $61.3 \%$ ) completed the NEO FFI personality questionnaire. Five years later, they filled in the scale of life events ISV and various questionnaires about internalising and externalising symptoms. To achieve the objective, stepwise regression analyses were performed. Results: Neuroticism, contentiousness, number of negative events and involvement predicted the internalising symptoms, while low agreeableness, neuroticism, negative and controllable life events and involvement predicted the externalising symptoms. All the effects of life events on psychopathology remained significant when personality was controlled for, except for controllable life events. Discussion: The results indicate that the effect of life events on psychopathology is not produced only by the influence of personality, but these have a direct impact on the development of psychopathology.

Keywords: personality, life events, internalising and externalising psychopatology.

\section{Introducción}

\section{Eventos vitales: concepto y tipologías}

Cuando se habla de eventos vitales negativos, es importante diferenciar entre eventos incontrolables y controlables. Los eventos vitales incontrolables son eventos cuya ocurrencia no depende del comportamiento de las personas (por ejemplo, la muerte de un ser querido), mientras que los eventos vitales controlables son potencialmente dependientes del comportamiento del propio sujeto (por ejemplo, despido laboral).

Por lo tanto, aunque durante bastante tiempo se pensó que la presencia de eventos vitales negativos a lo largo de la vida ocurría de forma totalmente aleatoria, esta creencia es errónea: no todos los eventos vitales ocurren de forma azarosa. (Saudino, Pedersen, Lichtenstein, McClearn y Plomin, 1997). De hecho, el número de eventos vitales reportados por una persona en distintos periodos de su vida presentan una relación significativa.

Esta idea recibe apoyo de los estudios de genética cuantitativa, los cuales muestran que en la ocurrencia de los eventos vitales incontrolables afectarían principalmente los factores ambientales, mientras que en el caso de la ocurrencia de los eventos vitales controlables o personales serían los factores genéticos los que explicarían gran parte del fenotipo (Kendler y Greenspan, 2006; Kendler y Neale, 1993; McGue y Bouchard, 1991; Plomin y Lichtenstein, 1990).

Cuando se intentan analizar las causas de la ocurrencia de los eventos vitales desde un punto de vista genético-epidemiológico se encuentra que los eventos vitales que se dan a lo largo de la vida muestran una correlación significativa entre pares de gemelos y está correlación es significativamente mayor en gemelos monozigóticos que en dizigóticos (Plomin, DeFries y Loehlin, 1997). Por tanto, resulta posible que determinados factores psicológicos, como la per- 
sonalidad, que están influenciados a su vez por factores genéticos, afecten a la posibilidad de experimentar eventos vitales negativos en mayor medida (Kendler y Greenspan, 2006).

Además, el número de eventos vitales se puede predecir a partir de otras características personales que se han asociado a su vez con la personalidad, como el apoyo social (Kendler, 1997; Kessler, Kendler, Heath, Neale y Eaves, 1992; Lin, Dean y Ensel, 2013), el consumo de drogas (Sümer, 2003; Tsuang, Boor y Fleming, 1985) o el nivel socio-económico (Kessler, 1979).

Entender la ocurrencia de los eventos vitales en sí mismos tiene especial relevancia ya que ese estrés ambiental suele manifestarse en forma de alteraciones psicopatológicas. Las aproximaciones genéticas pueden proporcionar información valiosa sobre la naturaleza de estas relaciones entre los sujetos y sus ambientes.

\section{Personalidad y eventos vitales}

Claramente, los factores genéticos no actúan directamente sobre ambientes específicos. La influencia genética en los eventos vitales se explica porque el medio no es independiente del individuo. Cada persona juega un rol activo en la creación de sus propios ambientes. Por tanto, estas influencias genéticas en los eventos vitales se dan, posiblemente, a través de la personalidad. De hecho, la influencia genética en los rasgos de personalidad es un hecho demostrado, del mismo modo que lo es la influencia de la personalidad en ciertos tipos de eventos vitales (Eaves, Eysenck y Martin, 1989). De aquí se deriva que los rasgos de personalidad afectan a cómo las personas se relacionan con sus ambientes y, como resultado, contribuyen a los eventos que se experimentan a lo largo de la vida. Además, numerosos estudios han encontrado que existe una relación bidireccional entre las situaciones y los individuos y que los individuos tienden a elegir situaciones o actividades que reflejan su propia personalidad (Diener, Larsen y Emmons, 1984).

Varios autores han investigado específicamente la relación entre rasgos concretos de personalidad y los eventos vitales. Mientras que el neuroticismo predice significantemente la presencia de eventos vitales negativos y la calidad de las relaciones interpersonales (Kendler y Greenspan, 2006); la extraversión, por el contrario, predispone a la ocurrencia de eventos vitales positivos (Magnus, Diener, Fujita y Pavot, 1993). Por lo que respecta a la apertura a la experiencia (O), esta dimensión de personalidad se asociaría tanto con los eventos vitales negativos como con los positivos (Headey y Wearing, 1989; Saudino y cols., 1997).

Otros autores, en cambio, encontraron que los acontecimientos vitales negativos poco controlables predecían mayores niveles de neuroticismo, mientras que estos eran, a su vez, predichos por la alta apertura a la experiencia y la baja amabilidad. No obstante, las relaciones son poco robustas y son los mismos autores los que concluyen que la ocurrencia de este tipo de eventos depende mucho más de factores ajenos a la persona que de la propia personalidad (Kandler, Bleidorn, Riemann, Angleitner y Spinath, 2012). Por otra parte, niveles altos de neuroticismo y de apertura a la experiencia, unidos a niveles bajos de amabilidad, aumentan la posibilidad de experimentar eventos vitales controlables (Kandler y cols., 2012). Moya y cols. (2015) también encontraron que los eventos controlables estaban predichos por niveles altos de extraversión y niveles bajos de amabilidad y responsabilidad, apoyando así la diferenciación entre ambas tipologías.

Por lo que respecta a la afectación percibida, aquellas personas con niveles elevados de neuroticismo y psicoticismo tenderían a percibir los acontecimientos vitales como más estresantes (Afshar y cols., 2015; Kardum y Krapić, 2001), mientras que la alta extraversión actuaría como un protector ante el estrés derivado de los eventos vitales y se relacionaría con un 
mayor número de eventos vitales positivos experimentados, menor grado de impacto y mayor bienestar subjetivo (Graziano, Feldesman y Rahe, 1985).

\section{Psicopatología y eventos vitales}

La presencia de estos eventos vitales negativos o estresantes contribuye al desarrollo y al inicio de sintomatología psicopatológica. Esta sintomatología se puede dividir en sintomatología internalizante (ansiedad, problemas afectivos, miedos y fobias) y sintomatología externalizante (conductas disruptivas y consumo de sustancias entre otros) (Krueger y Markon, 2006).

Estudios previos han encontrado que los eventos vitales negativos, tanto controlables como incontrolables, se relacionan con sintomatología de tipo internalizante y externalizante (Flouri y Kallis, 2011; Lyons, Huebner, Hills y Van Horn, 2013).

\section{Personalidad y psicopatología: el papel de los eventos vitales}

La relación entre las distintas dimensiones de personalidad y ambos tipos de sintomatología psicopatológica muestra un cierto grado de especificidad a través de distintos estudios llevados a cabo. De este modo, los trastornos relacionados con depresión o ansiedad se relacionarían más con niveles elevados de neuroticismo, mientras que el abuso de sustancias se relacionaría con desinhibición y baja amabilidad (Kotov, Gamez, Schmidt y Watson, 2010). Siguiendo esta misma línea, otros metanálisis han encontrado que el alto afecto negativo, la irresponsabilidad, la desinhibición y la baja amabilidad se relacionan con síntomas y trastornos de tipo externalizante (Maclaren, Fugelsang, Harrigan y Dixon, 2011; Malouff, Thorsteinsson, Rooke y Schutte, 2007). Por lo tanto, la sintomatología de tipo internalizante se asocia con neuroticismo, mientras que la sintomatología externalizante se asocia con baja amabilidad y baja responsabilidad (Mezquita y cols., 2015).

En vista de estos estudios previos, la personalidad y los eventos vitales negativos interactúan entre sí y esto facilita la predicción de sintomatología psicopatológica. La personalidad se asociaría con la probabilidad de experimentar eventos vitales en mayor o menor medida, a la vez que estos eventos vitales podrían influir en cambios en la personalidad, originando así transacciones persona-ambiente relacionadas con el tipo de psicopatología expresada.

Siguiendo esta línea, varios autores han encontrado que la responsabilidad y el neuroticismo moderan el afrontamiento de los eventos vitales negativos e incontrolables. No obstante, el neuroticismo, a su vez, amplifica los efectos de estos eventos en relación a la psicopatología desarrollada (van Zuiden y cols., 2011; Widiger y Smith, 2008). Por otra parte, niveles elevados de neuroticismo se relacionan con una mayor percepción y número de eventos vitales negativos y, por tanto, con mayores niveles de estrés, mayor afecto negativo y mayor riesgo de experimentar psicopatología de tipo internalizante (Mandelli y cols., 2015). Por otra parte, aquellos con niveles elevados de extraversión tienden a experimentar más cantidad de EV debido a la búsqueda de novedad y de emociones intensas, aumentando a su vez la probabilidad de experimentar sintomatología externalizante (Viruela, Camacho, Mezquita y Moya, 2009). El objetivo del presente trabajo fue evaluar en qué medida los eventos vitales influyen en el desarrollo de la psicopatología internalizante y externalizante, una vez se controla el efecto de la personalidad.

Las hipótesis planteadas fueron: 1) niveles elevados de neuroticismo se asociarán con mayor sintomatología internalizante, mientras que niveles elevados de impulsividad se relacio- 
narán con sintomatología externalizante; 2) la ocurrencia de eventos vitales predecirá la aparición de psicopatología; 3) la controlabilidad de los eventos vitales predecirá la aparición de psicopatología (relación dependiente de personalidad), mientras que la incontrolabilidad de los eventos vitales predecirá la aparición de psicopatología (relación independiente de personalidad) y 4) la personalidad influiría en la afectación percibida y esta, a su vez, en el desarrollo de psicopatología.

\section{Método}

\section{Participantes}

La muestra está compuesta por 336 adultos jóvenes (61,3\% eran mujeres), con una media de edad de 21,68 años $(S D=3,85)$. El rango de edad de la muestra va de los 18 a los 50 años.

\section{Procedimiento}

Durante el año 2004 los participantes completaron el cuestionario de personalidad NEOFFI. Cinco años más tarde cumplimentaron la escala de eventos vitales ISV y varios cuestionarios sobre síntomas internalizantes (BDI, PSWQ, ACQ y FQ) y externalizantes (APD, LSRP, CPQ, AUDIT y SOGS). Todos los instrumentos se administraron de forma presencial con una remuneración de 20 euros en el 2004 y de 30 euros en el 2009. Los participantes fueron informados del proyecto, de la confidencialidad con la que se tratarían sus datos y de la posibilidad de abandonar la investigación en el momento que quisiesen.

\section{Instrumentos}

Personalidad:

- El NEO-FFI (Costa y McCrae, 1992) es un cuestionario que contiene 60 ítems, que, a través de una escala Likert de 5 puntos $(0=$ totalmente en desacuerdo y $5=$ totalmente de acuerdo) evalúa las cinco dimensiones de personalidad basadas en los cinco grandes: neuroticismo $(\mathrm{N})$, extraversión $(\mathrm{E})$, apertura a la experiencia $(\mathrm{O})$, amabilidad (A) y responsabilidad (C).

\section{Eventos vitales:}

- El inventario de eventos vitales (ISV, construido por el grupo de investigación IDAP en el 2009) consta de 70 eventos vitales estresantes de distinta naturaleza. En el presente estudio se tuvieron en cuenta solo aquellos que se habían experimentado en el último año, así como el grado de afectación asociado a cada evento vital (-4 = extremadamente negativo a $+4=$ extremadamente positivo). Los ítems de este cuestionario se sometieron al juicio de expertos para determinar la controlabilidad o incontrolabilidad de los distintos sucesos vitales. 
Sintomatología internalizante:

- El inventario de preocupación de Pensilvania (PSQW; Nuevo, Montorio y Ruiz, 2002) es un autoinforme de 16 ítems que evalúa la tendencia general a preocuparse mediante una escala Likert de 5 puntos ( $0=$ nada y $4=$ mucho).

- El inventario de depresión de Beck-II (BDI-II; Sanz, García-Vera, Espinosa, Fortún y Vázquez, 2005) consta de 21 ítems con 4 alternativas de respuesta basados en los criterios diagnósticos del DSM-IV. Permite evaluar la presencia y gravedad de la sintomatología depresiva.

- La escala de fobia social SP es una escala de 5 ítems perteneciente al cuestionario de miedos (FQ; Marks y Mathews, 1979) que permite evaluar la evitación a determinadas situaciones sociales a partir de una escala. Las respuestas se dan en un rango que va de 0 (no lo evitaría) a 8 (siempre lo evito).

- El cuestionario de cogniciones agorafóbicas (ACQ; Chambless, Caputo, Bright y Gallagher, 1984) evalúa la frecuencia y el tipo de pensamientos asociados a los ataques de pánico y agorafobia, así como las consecuencias negativas de la ansiedad a través de 15 ítems de tipo Likert ( $1=$ nunca y $5=$ siempre $)$.

Sintomatología externalizante:

- El cuestionario de identificación de los transtornos debidos al consumo de alcohol (AUDIT; Babor y Higgins-Biddle, 2001) es un autoinforme de 10 ítems que, a través de una escala tipo Likert, evalúa el consumo de alcohol, las conductas de dependencia y los problemas derivados del consumo durante el último año.

- El cuestionario de problemas asociados al consumo de cannabis (CPQ; Copeland, Gilmour, Gates y Swift, 2005) mide los problemas asociados al consumo de cannabis. Consta de 27 ítems de respuesta dicotómica sí/no, en los que los participantes responden acerca de sus experiencias derivadas del consumo del cannabis en los últimos tres meses.

- La escala de psicopatía de Levenson (LSRP; Lynam, Whiteside y Jones, 1999) consta de 26 ítems de tipo Likert con 4 puntos ( 1 = muy en desacuerdo y $4=$ muy de acuerdo). Esta escala evalúa diferentes síntomas asociados a la psicopatía como orientación manipulativa, egoísmo e insensibilidad.

- El cuestionario de juego de South Oaks (sogs; Echeburúa, Báez, FernéndezMontalvo y Pérez, 1994) es un cuestionario de 20 ítems de tipo dicotómico que permite evaluar la dependencia al juego a través de preguntas acerca de las conductas de juego, las fuentes de obtención de dinero y las emociones implicadas.

- Para evaluar los síntomas del trastorno antisocial de la personalidad se utilizó una escala de 7 ítems verdadero/falso del examen internacional de trastornos de la personalidad (IPDE, López-Ibor, Pérez-Urdániz y Rubio, 1996).

\section{Análisis}

Mediante el uso del software SPSS versión 23, se realizaron análisis descriptivos de la muestra total, para hombres y para mujeres; posteriormente, se calcularon las diferencias de 
sexo mediante una prueba $t$ y también el tamaño del efecto de estas diferencias a través de la $d$ de Cohen (1992), mediante la calculadora online del tamaño del efecto (Becker, 1999).

Mediante este mismo software se realizó un análisis factorial con los distintos instrumentos de sintomatología internalizante y externalizante para obtener un factor de internalización y otro de externalización. Los factores resultantes se guardaron en la base de datos para su posterior análisis.

Posteriormente, se obtuvieron las correlaciones entre las distintas variables y se realizaron análisis de regresión lineal simple por pasos, siendo las variables dependientes la sintomatología internalizante y externalizante. Tras controlar el efecto de la edad y el género, se introdujeron las variables predictoras. En el segundo paso se introdujo el número de eventos vitales positivos, negativos y totales del último año, los eventos vitales controlables e incontrolables y la afectación. Finalmente, en el último paso se introdujeron las dimensiones de personalidad.

\section{Resultados}

\section{Análisis descriptivos}

Los análisis descriptivos aparecen en la tabla 1. Las mujeres puntuaron significativamente más alto en neuroticismo y en amabilidad que los hombres; mientras que los hombres mostraron niveles más elevados de sintomatología externalizante.

Tabla 1

Análisis descriptivos de la muestra total y diferenciando por sexo

\begin{tabular}{lccccc}
\hline & $\begin{array}{c}\text { Muestra total } \\
(\mathrm{N}=336) \\
\mathrm{M}(\mathrm{SD})\end{array}$ & $\begin{array}{c}\text { Hombres } \\
(\mathrm{N}=129) \\
\mathrm{M}(\mathrm{SD})\end{array}$ & $\begin{array}{c}\text { Mujeres } \\
(\mathrm{N}=204) \\
\mathrm{M}(\mathrm{SD})\end{array}$ & $t$ & $d$ \\
\hline Personalidad & & & & \\
N & $19,45(7,72)$ & $16,67(7,27)$ & $21,21(7,49)$ & $-5,44^{* * *}$ & 0,61 \\
E & $31,10(6,40)$ & $30,92(6,18)$ & $31,22(6,54)$ & $-0,41$ & 0,05 \\
O & $29,05(6,13)$ & $28,63(6,48)$ & $29,31(5,90)$ & $-0,99$ & 0,11 \\
A & $33,06(6,07)$ & $31,12(6,52)$ & $34,29(5,44)$ & $-4,81^{* * *}$ & 0,53 \\
C & $29,26(7,35)$ & $28,50(7,38)$ & $29,74(7,30)$ & $-1,50$ & 0,17 \\
\hline EV incontrolables & $1,00(1,02)$ & $0,93(0,98)$ & $1,03(1,05)$ & $-0,86$ & 0,10 \\
\hline EV controlables & $3,00(2,35)$ & $2,97(2,04)$ & $3,00(2,50)$ & $-0,14$ & 0,02 \\
\hline EV total & $4,55(2.94)$ & $4,43(2,66)$ & $4,60(3,10)$ & $-0,50$ & 0,06 \\
\hline EV positivos & $2,07(1,78)$ & $2,05(1,71)$ & $2,07(1,84)$ & $-0,11$ & 0,01 \\
\hline EV negativos & $2,26(2,10)$ & $2,14(2,06)$ & $2,33(2,14)$ & $-0,80$ & 0,09 \\
\hline Afectación positiva & $5,99(5,69)$ & $5,95(5,75)$ & $6,03(5,70)$ & $-0,13$ & 0,01 \\
\hline
\end{tabular}




\begin{tabular}{lccccc}
\hline & $\begin{array}{c}\text { Muestra total } \\
(\mathrm{N}=336) \\
\mathrm{M}(\mathrm{SD})\end{array}$ & $\begin{array}{c}\text { Hombres } \\
(\mathrm{N}=129) \\
\mathrm{M}(\mathrm{SD})\end{array}$ & $\begin{array}{c}\text { Mujeres } \\
(\mathrm{N}=204) \\
\mathrm{M}(\mathrm{SD})\end{array}$ & $t$ & $d$ \\
\hline Afectación negativa & $5,15(5,46)$ & $4,72(4,87)$ & $5,42(5,81)$ & $-1,14$ & 0,13 \\
\hline Factor internalizante & $-0,00(0,89)$ & $-0,11(0,86)$ & $0,06(0,90)$ & $-1,83$ & 0,21 \\
\hline $\begin{array}{l}\text { Factor } \\
\text { externalizante }\end{array}$ & $0,00(0,81)$ & $0,35(0,94$ & $-0,22(0,63)$ & $6,56^{* * *}$ & 0,71 \\
\hline
\end{tabular}

Nota. Los valores de la $d$ de Cohen $0,20,0,50$ y 0,80 corresponden a efectos pequeños, medianos y grandes respectivamente (Cohen, 1992). ${ }^{*} p<0,05 .{ }^{* *} p<0,01 .{ }^{* * *} p<0,001$.

\section{Análisis de regresión}

Los análisis de regresión mostraron que el neuroticismo, la responsabilidad, el número de eventos negativos y la afectación predijeron la sintomatología internalizante; mientras que la baja amabilidad, el neuroticismo, los eventos negativos, el número total de EV, EV controlables y la afectación predijeron la sintomatología externalizante. Todos los efectos de los eventos vitales sobre la psicopatología se mantuvieron significativos cuando se controló la personalidad, a excepción de los eventos vitales controlables (véase la tabla 2).

Tabla 2

Análisis de regresión

\begin{tabular}{|c|c|c|c|c|c|c|c|c|c|c|}
\hline & \multirow[b]{3}{*}{$\begin{array}{l}\text { Variables } \\
\text { independientes }\end{array}$} & \multicolumn{4}{|c|}{ Variables dependientes } & \multirow[b]{3}{*}{$\begin{array}{c}\text { Variables } \\
\text { independientes }\end{array}$} & \multicolumn{4}{|c|}{ Variables dependientes } \\
\hline & & \multicolumn{2}{|c|}{$\begin{array}{c}\text { Factor } \\
\text { internalizante }\end{array}$} & \multicolumn{2}{|c|}{$\begin{array}{c}\text { Factor } \\
\text { externalizante }\end{array}$} & & \multicolumn{2}{|c|}{$\begin{array}{c}\text { Factor } \\
\text { internalizante }\end{array}$} & \multicolumn{2}{|c|}{$\begin{array}{c}\text { Factor } \\
\text { externalizante }\end{array}$} \\
\hline & & $\beta$ & $\Delta R^{2}$ & $\beta$ & $\Delta R^{2}$ & & $\beta$ & $\Delta R^{2}$ & $\beta$ & $\Delta R^{2}$ \\
\hline \multirow{2}{*}{1} & Género & 0,10 & $0,02^{* *}$ & $-0,34^{* * *}$ & $0,12^{* * *}$ & Género & 0,10 & $0,02^{* *}$ & $-0,34^{* * *}$ & $0,12^{* * *}$ \\
\hline & Edad & $-0,14^{*}$ & & $-0,08$ & & Edad & $-0,14^{*}$ & & $-0,08$ & \\
\hline & Género & 0,09 & $0,07^{* * *}$ & $-0,35^{* * *}$ & $0,14^{* * *}$ & Género & 0,09 & $0,03^{* *}$ & $-0,35^{\star * *}$ & $0,13^{* * *}$ \\
\hline \multirow{2}{*}{2} & Edad &,$- 14^{\star *}$ & & $-0,08$ & & Edad & $-0,13^{*}$ & & $-0,07$ & \\
\hline & EV negativo & $0,22^{* * *}$ & & $0,16^{* *}$ & & EV total & 0,10 & & $0,14^{* *}$ & \\
\hline & EV positivo & $-0,10$ & & 0,02 & & & & & & \\
\hline \multirow{9}{*}{3} & Género & $-0,04$ & $0,31^{* * *}$ & $-0,33^{* * *}$ & $0,22^{* * *}$ & Género & $-0,03$ & $0,30^{* * *}$ & $-0,33^{* * *}$ & $0,22^{* * *}$ \\
\hline & Edad & $-0,15^{\star *}$ & & $-0,04$ & & Edad & $-0,13^{* *}$ & & $-0,04$ & \\
\hline & EV negativo & $0,16^{* *}$ & & $0,12^{*}$ & & EV total & 0,08 & & $0,11^{*}$ & \\
\hline & EV positivo & $-0,06$ & & 0,04 & & & & & & \\
\hline & $\mathrm{N}$ & $0,49^{* * *}$ & & $0,16^{*}$ & & N & $0,50^{* * *}$ & & $0,16^{* *}$ & \\
\hline & E & $-0,02$ & & 0,09 & & E & $-0,03$ & & 0,08 & \\
\hline & $\mathrm{O}$ & $-0,02$ & & $-0,01$ & & $\mathrm{O}$ & $-0,03$ & & $-0,01$ & \\
\hline & $A$ & $-0,10$ & & $-0,22^{* * *}$ & & A & $-0,10$ & & $-0,22^{\star * *}$ & \\
\hline & C & $0,12^{*}$ & & $-0,09$ & & C & $0,12^{*}$ & & $-0,09$ & \\
\hline
\end{tabular}




\begin{tabular}{|c|c|c|c|c|c|c|c|c|c|c|}
\hline & \multirow[b]{3}{*}{$\begin{array}{l}\text { Variables } \\
\text { independientes }\end{array}$} & \multicolumn{4}{|c|}{ Variables dependientes } & \multirow[b]{3}{*}{$\begin{array}{c}\text { Variables } \\
\text { independientes }\end{array}$} & \multicolumn{4}{|c|}{ Variables dependientes } \\
\hline & & \multicolumn{2}{|c|}{$\begin{array}{c}\text { Factor } \\
\text { internalizante }\end{array}$} & \multicolumn{2}{|c|}{$\begin{array}{c}\text { Factor } \\
\text { externalizante }\end{array}$} & & \multicolumn{2}{|c|}{$\begin{array}{c}\text { Factor } \\
\text { internalizante }\end{array}$} & \multicolumn{2}{|c|}{$\begin{array}{c}\text { Factor } \\
\text { externalizante }\end{array}$} \\
\hline & & $\beta$ & $\Delta R^{2}$ & $\beta$ & $\Delta R^{2}$ & & $\beta$ & $\Delta \mathrm{R}^{2}$ & $\beta$ & $\Delta R^{2}$ \\
\hline \multirow{2}{*}{1} & Género & 0,10 & $0,02^{* * *}$ & $-0,34^{* * *}$ & $0,12^{* * *}$ & Género & 0,10 & $0,02^{* *}$ & $-0,34^{* * *}$ & $0,12^{* * *}$ \\
\hline & Edad & $-0,14^{*}$ & & $-0,08$ & & Edad & $-0,14^{*}$ & & $-0,08$ & \\
\hline \multirow{4}{*}{2} & Género & 0,08 & $0,10^{* * *}$ & $-0,35^{* \star *}$ & $0,14^{* \star *}$ & Género & 0,09 & $0,03^{*}$ & $-0,35^{* * *}$ & $0,13^{* * *}$ \\
\hline & Edad &,$- 14^{* *}$ & & $-0,08$ & & Edad & $-0,13^{*}$ & & $-0,08$ & \\
\hline & $\begin{array}{l}\text { Afectación } \\
\text { negativa }\end{array}$ & $0,28^{* * *}$ & & $0,16^{* *}$ & & Incontrolable & 0,03 & & 0,06 & \\
\hline & $\begin{array}{l}\text { Afectación } \\
\text { positiva }\end{array}$ & $-0,10$ & & $-0,01$ & & Controlable & 0,10 & & $0,13^{\star *}$ & \\
\hline \multirow{9}{*}{3} & Género & $-0,04$ & $0,35^{* * *}$ & $-0,33^{* * *}$ & $0,22^{* \star *}$ & Género & $-0,03$ & $0,30^{* * *}$ & $-0,33^{* * *}$ & $0,22^{* * *}$ \\
\hline & Edad & $-0,15^{\star *}$ & & $-0,05$ & & Edad & $-0,13^{* *}$ & & $-0,04$ & \\
\hline & $\begin{array}{l}\text { Afectación } \\
\text { negativa }\end{array}$ & $0,21^{* * *}$ & & $0,12^{*}$ & & Incontrolable & 0,03 & & 0,06 & \\
\hline & $\begin{array}{l}\text { Afectación } \\
\text { positiva }\end{array}$ & $-0,06$ & & 0,01 & & Controlable & 0,06 & & 0,09 & \\
\hline & $\mathrm{N}$ & $0,49^{* * *}$ & & $0,16^{*}$ & & $\mathrm{~N}$ & $0,50^{* * *}$ & & $0,15^{*}$ & \\
\hline & $E$ & $-0,02$ & & 0,09 & & E & $-0,03$ & & 0,08 & \\
\hline & 0 & $-0,02$ & & 0,01 & & 0 & $-0,03$ & & $-0,01$ & \\
\hline & A & $-0,09$ & & $-0,22^{* * *}$ & & A & $-0,10$ & & $-0,22^{* * *}$ & \\
\hline & C & $0,12^{*}$ & & $-0,09$ & & C & $0,11^{*}$ & & $-0,09$ & \\
\hline
\end{tabular}

Nota. ${ }^{*} p<0.05^{* *} p<0.01{ }^{* * *} p<0.001$

\section{Discusión}

El presente estudio tenía como objetivo principal evaluar en qué medida los eventos vitales influyen en el desarrollo de la psicopatología internalizante y externalizante, una vez se controla el efecto de la personalidad.

En lo relativo a la relación de personalidad con patología, en este estudio se ha encontrado que, de forma consistente con estudios previos, niveles elevados de neuroticismo se relacionan con mayor sintomatología internalizante, posiblemente porque el neuroticismo se asocia a mayores niveles de estrés y de afecto negativo. Mientras que características de impulsividad y deshinibición, baja amabilidad en este caso, se asocian con sintomatología externalizante (Kotov y cols., 2010; Mezquita y cols., 2015) .

Por lo que respecta a los eventos vitales, su ocurrencia predice la aparición de psicopatología. Si bien los eventos vitales negativos se relacionan con sintomatología tanto internalizante como externalizante (Flouri y Kallis, 2011; Krueger y Markon, 2006), los eventos vitales totales experimentados en el último año solo se asocian con sintomatología de tipo externalizante. Esto podría deberse a que, al referirse al número total de eventos vitales, se incluyen tanto aquellos que generan distrés, estrés negativo, como aquellos que generan eutres, estrés positivo. Además, tampoco se tiene en cuenta la gravedad del evento vital experiementado.

Los eventos vitales de tipo controlable también predicen la aparición de sintomatología psicopatológica pero, en este caso, la relación es dependiente de la personalidad, ya que el efecto de los eventos vitales controlables sobre la sintomatología externalizante no permanece significativo cuando se controla la personalidad (Kandler y cols., 2012), apoyando la hipó- 
tesis de que la ocurrencia de eventos vitales controlables depende potencialmente del comportamiento y la personalidad del sujeto (Kendler y Greenspan, 2006). Por otra parte, totalmente en contra de lo esperado, no se encuentra ninguna relación significativa entre los eventos vitales incontrolables y la psicopatología, posiblemente porque la afectación derivada del evento vital y la interpretación del mismo como positivo o negativo es más relevante que la incontrolabilidad del propio evento.

Finalmente, la personalidad influye en la afectación percibida y esta, a su vez, en el desarrollo de psicopatología. La afectación percibida se relaciona con el desarrollo de psicopatología; una mayor percepción negativa genera mayor estrés y formas de coping menos adaptativas, aumentando así el riesgo de experimentar psicopatología (Mandelli y cols., 2015). No obstante, la afectación percibida está a su vez influida por la personalidad, ya que, al controlar el efecto de esta, la magnitud de la asociación entre afectación y psicopatología se reduce (Kandler y cols., 2012).

Como conclusión, la relación entre ocurrencia, grado de afectación y controlabilidad de los eventos vitales y el desarrollo de la psicopatología se debe a un impato ambiental, ya que las relaciones entre eventos vitales y psicopatología se mantienen aún cuando se controla la personalidad; pero también es resultado de una personalidad vulnerable, ya que, en el caso de los eventos controlables, desaparece la relación cuando se controla el efecto de la personalidad $y$, en el resto de asociaciones entre eventos vitales y psicopatología, la magnitud de las mismas disminuye al controlar la personalidad.

\section{Referencias bibliográficas}

Afshar, H., Roohafza, H. R., Keshteli, A. H., Mazaheri, M., Feizi, A. y Adibi, P. (2015). The association of personality traits and coping styles according to stress level. Journal of Research in Medical Sciences: The Official Journal of Isfahan University of Medical Sciences, 20, 353-358.

Babor, T. y Higgins-Biddle, J. (2001). AUDIT: the alcohol use disorders identification test: guidelines for use in primary health care. Geneva, Switzerland: World Health Organization.

Chambless, D. L., Caputo, G. C., Bright, P. y Gallagher, R. (1984). Assessment of fear of fear in agoraphobics: The Body Sensations Questionnaire and the Agoraphobic Cognitions Questionnaire. Journal of Consulting and Clinical Psychology, 52, 1090-1097.

Copeland, J., Gilmour, S., Gates, P. y Swift, W. (2005). The Cannabis Problems Questionnaire: factor structure, reliability, and validity. Drug and Alcohol Dependence, 80, 313-319.

Costa, P. T. y McCrae, R. R. (1992). Four ways five factors are basic. Personality and Individual Differences, 13, 653-665.

Flouri, E. y Kallis, C. (2011). Adverse life events and mental health in middle adolescence. Journal of Adolescence, 34, 371-377.

Graziano, W. G., Feldesman, A. B. y Rahe, D. F. (1985). Extraversion, social cognition, and the salience of aversiveness in social encounters. Journal of Personality and Social Psychology, 49, 971-980.

Kandler, C., Bleidorn, W., Riemann, R., Angleitner, A. y Spinath, F. M. (2012). Life events as environmental States and genetic traits and the role of personality: a longitudinal twin study. Behavior Genetics, 42, 57-72.

Kardum, I. y Krapic, N. (2001). Personality traits, stressful life events, and coping styles in early adolescence. Personality and Individual Differences, 30, 503-515.

Kendler, K. (1997). Social support: a genetic-epidemiologic analysis. American Journal of Psychiatry, 154, 322-329. 
Kendler, K. y Greenspan, R. (2006). The nature of genetic influences on behavior: Lessons from "simpler" organisms. American Journal of Psychiatry, 163, 1683-1694.

Kessler, R. (1979). Stress, social status, and psychological distress. Journal of Health and Social Behavior, 20, 259-272.

Kotov, R., Gamez, W., Schmidt, F. y Watson, D. (2010). Linking "big" personality traits to anxiety, depressive, and substance use disorders: a meta-analysis. Psychological Bulletin, 136, 768-821.

Krueger, R. F. y Markon, K. E. (2006). Reinterpreting comorbidity: a model-based approach to understanding and classifying psychopathology. Annual Review of Clinical Psychology, 2, 111-133.

Lynam, D. R., Whiteside, S., y Jones, S. (1999). Self-reported psychopathy: A validation study. Journal of Personality Assessment, 73, 110-132.

Lyons, M. D., Huebner, E. S., Hills, K. J. y Van Horn, M. L. (2013). Mechanisms of change in adolescent life satisfaction: a longitudinal analysis. Journal of School Psychology, 51, 587-598.

Maclaren, V. V, Fugelsang, J. A., Harrigan, K. A. y Dixon, M. J. (2011). The personality of pathological gamblers: a meta-analysis. Clinical Psychology Review, 31, 1057-1067.

Magnus, K., Diener, E., Fujita, F. y Pavot, W. (1993). Extraversion and neuroticism as predictors of objective life events: A longitudinal analysis. Journal of Personality and Social Psychology, 65, 1046-1053.

Malouff, J., Thorsteinsson, E., Rooke, S. y Schutte, N. (2007). Alcohol Involvement and the FiveFactor Model of Personality: A Meta-Analysis. Journal of Drug Education, 37, 277-294.

Mandelli, L., Nearchou, F. A., Vaiopoulos, C., Stefanis, C. N., Vitoratou, S., Serretti, A. y Stefanis, N. C. (2015). Neuroticism, social network, stressful life events: association with mood disorders, depressive symptoms and suicidal ideation in a community sample of women. Psychiatry Research, 226, 38-44.

Marks, I. M., y Mathews, A. M. (1979). Brief standard self-rating for phobic patients. Behaviour Research and Therapy, 17, 263-267.

Mezquita, L., Ibáñez, M. I., Villa, H., Fañanás, L., Moya-Higueras, J. y Ortet, G. (2015). Fivefactor model and internalizing and externalizing syndromes: A 5-year prospective study. Personality and Individual Differences, 79, 98-103.

Nuevo, R., Montorio, I. y Ruiz, M. Á. (2002). Aplicabilidad del Inventario de Preocupación de Pensilvania (PSWQ) a la población de edad avanzada. Ansiedad y Estrés, 8, 157-172.

Plomin, R., DeFries, J. C. y Loehlin, J. C. (1977). Genotype-environment interaction and correlation in the analysis of human behavior. Psychology Bulletin, 84, 309-322.

Plomin, R. y Lichtenstein, P. (1990). Genetic influence on life events during the last half of the life span. Psychology Aging, 5, 25-30.

Sanz, J., García-Vera, M. P., Espinosa, R., Fortún, M. y Vázquez, C. (2005). Adaptación española del Inventario para la Depresión de Beck-II (BDI-II). Propiedades psicométricas en pacientes con trastornos psicológicos. Clínica y Salud, 16, 121-142.

Saudino, K. J., Pedersen, N. L., Lichtenstein, P., McClearn, G. E. y Plomin, R. (1997). Can personality explain genetic influences on life events? Journal of Personality and Social Psychology, 72, 196-206.

Sümer, N. (2003). Personality and behavioral predictors of traffic accidents: testing a contextual mediated model. Accident Analysis \& Prevention, 35(6), 949-964.

Viruela, A., Camacho, L., Mezquita, L. y Moya, J. (2009). Personalidad y sucesos vitales negativos en la adolescencia. Fòrum de recerca, 15, 261-272. 